\title{
Design of a minimum weight/space/energy consumption instrumentation system
}

\author{
Florian Mertl, Renaud Urli \\ Airbus Helicopters Deutschland GmbH, Industriestrasse 4, 86607 Donauwörth, Germany \\ Florian.Mertl@airbus.com \\ Renaud.Urli@airbus.com
}

\begin{abstract}
Research platforms require flight test instrumentation systems fulfilling the difficult compromise between minimum weight, space, energy consumption and maximum flexibility combined with a high number of different measurement requirements.

This paper describes the challenges faced during the design of such an instrumentation system, the changes introduced in sequential iterations in the standard architectures of FTI (Flight Test Instrumentation) systems for large AC (aircraft) prototypes, as well as some limits met during the design.
\end{abstract}

Key words: FTI, Flight Test Instrumentation, Weight, Size, Consumption, Requirements Engineering, demonstrator, research

\section{Introduction}

Standard architectures of FTI installations are based on the different functions required in an FTI environment. Therefore each function (e. g. Time Service, Recording, Control) is covered by separate equipment. In addition, the requirement of serving an upmost flexible FTI platform which shall be capable of handling an increasing number of parameters leads to a certain amount of weight, size and of course power consumption of the FTI. Standard test aircraft can cope with non-optimized designs of FTI installations regarding those latter parameters but research platforms or aircraft demonstrators, depending on their mission like e.g. Urban Mobility, will demand a drastically reduced FTI footprint. Several methods shall be used to reach this goal, dealing with both technical and organizational aspects. Despite normal (standard) FTI installations, these contribution factors become high priority elements when designing new aircraft concepts or demonstrators for certain use cases like e. $g$. urban mobility.

\section{Weight}

Weight shall be seen as the most important parameter when building the architecture of a FTI System for a research aircraft. As the optimization of the Payload/AC Weight Ratio already in demonstrator phase is a key element while designing new aircraft concepts, it's obvious that $\mathrm{FTI}$ weight must also be reduced to a minimum. But even if this requirement sounds simple, it has a huge impact on the FTI design. Which steps could help to decrease the FTI weight?

First, do not use LRU (Line replaceable unit) trays. LRU trays offer quick installation/deinstallation of the equipment as well as easy access for maintenance and repair. Some trays even provide mechanical damping for protection of tape- or HDD-driven equipment. Removing the tray gives you the benefit of a considerable weight reduction on the one hand, but forces you to provide fix provisions for FTI in the AC's primary structure in the very early design stages of the aircraft.

Second, avoid cut-off connectors. It's obvious that a connection point with plug/receptacle and mechanical support is much heavier than the FTI cable itself in the same area. If sensors are used with pre-assembled cable directly connected at the sensor side, keep in mind to have the cable long enough to route direct from sensor to DAU (data acquisition unit).

As it can be seen in Fig $1,10 \mathrm{~cm}$ of a 4 wire sensor cable weighs $2.5 \mathrm{~g}$. Putting a cut-off connector - assuming a "light" connector type at this point, it will add a weight of $100 \mathrm{~g}$. As a consequence, suppressing cut-off connectors for an medium-sized FTI installation with 100200 lines will save up to $10-20 \mathrm{~kg}$. 
wired section vs. cut-off section

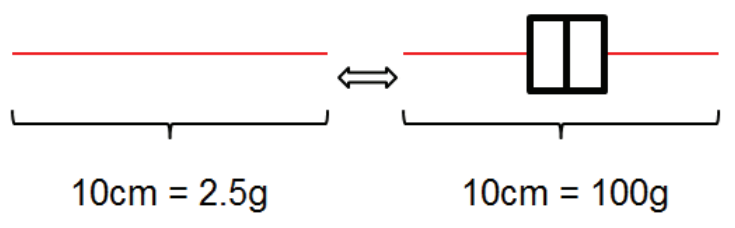

Fig 1 Comparison weight of cable and weight of connection point

Combine functions. As mentioned above, it's common to have one piece of equipment responsible for one function. By combining functions, it's possible to reduce the number of equipment (see Fig 2), ergo reduce weight. For instance, modern FTI systems offer a recording function in the DAU, which makes a separate recorder needless. To achieve this, it's mandatory to determine the necessary recording bandwidth in an early state in order to identify if this combination is possible.

Shift functions. Depending on the test mission, some functions are not needed on board (e. g. TOP Event) while others are required on ground (command and control). These functions shall be therefore covered by the FTI telemetry ground station. This allows the designer to remove the equipment from the aircraft. The constraint is to ensure that these functions can be handled by FTI telemetry ground station.

\section{Num. of Equipment}

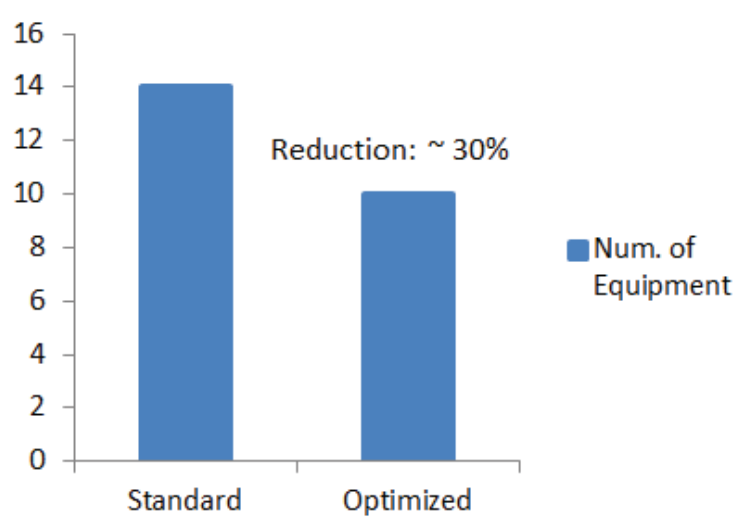

Fig 2 Number of equipment in a medium-sized FTI system; Standard architecture vs. optimized architecture

\section{Total Weight $[\mathrm{kg}]$}

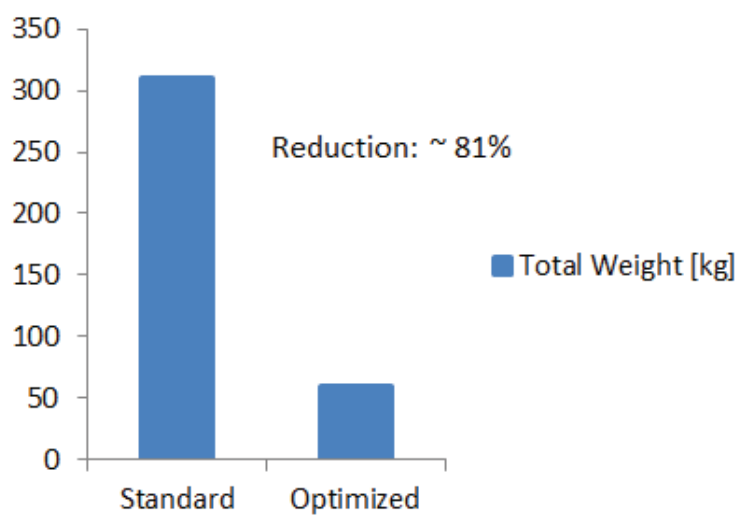

Fig 3 Total Weight of a medium-sized FTI system; Standard architecture vs. optimized architecture

\section{Size}

Succeeding in weight reduction is a good basis to focus on the next challenge, reducing the size of the FTI to a minimum. It has to be considered that there's always smaller equipment available on the market. Innvoative manufacturers offer new miniaturized DAU's providing higher channel density, more functionality, better performance etc. But in the present context, there will always be a compromise between risk and benefit. Risks to be considered are longer lead time, reduced support quality, late bug fixing, and integration testing issues, release cycle and cost. Accordingly, in most cases the challenge will be to reduce size with the already existing and available equipment.

Decentralize FTI. Usually FTI installations are intended to be installed and de-installed easily. Often a central rack is built in which all - or at least almost all - FTI equipment are installed. This rack is to be fitted in the seat rails and provides connector panels: hence quick to handle, move and maintain. The drawback of this setup is its size. Relocating the FTI equipment to places where free space is available and furthermore shorter cable routes to sensors and tapings are possible, offers size and cable weight reduction.

Avoid cut-off connectors. As mentioned above, cut-off connectors contribute not only to more weight but also to bigger size. Mechanical parts dedicated for FTI often have an impact on the structure design of the aircraft itself. Therefore it degrades the representativeness of the platform compared to the basic design. Substantiations have to be made in order to be able to transfer the obtained data into a qualifiable model. In addition keeping off the cut-off connectors means having a as direct as possible link 
between the signal source (sensor, bus) and the DAU.

Avoid spare cabling. To cope with changing demands during testing phases and campaigns, it is common that some spare cabling is foreseen. Doing that, it's possible to react in a flexible way to new needs when operating the aircraft. To fulfill the size reduction requirement, spare cables have to be avoided or, at least, reduced to an absolute minimum. It has to be considered that the success of this process is particularly depending on a disciplined requirement engineering phase. Furthermore, planning a dedicated connection to the bus tapping points from the aircraft systems suppresses duplicated bus distribution modules.

\section{Power Consumption}

Reducing the power consumed by FTI equipment is a process with limited opportunities nowadays.

It is essential to identify as exactly as possible the power required by $\mathrm{FTI}$. This postulation leads to a challenging requirement engineering process which has to take place very early, and eventually leads to the reduction of the number of equipment and hence to the power consumption.

Low power transducers like nanotechnology MEMS sensors are not to be easily integrated in current $\mathrm{FTI}$ systems as the conditioning equipment is not widely available yet.

Usage of new functions. Modern bus monitor modules in DAU's are capable of gathering data from specific signals (Arinc 429, Serial, Video) only when data are available on the bus. These modules enable a bandwidth reduction by processing and passing the data to the backplane only if an active signal is detected. This leads to an efficient bandwidth usage, improves the recording time as well as the time for data handling in post processing.

Implementation of wireless technology. Although instrumentation requirements are rarely fulfilled by current off-the-shelf wireless systems like IoT ones, their usage shall be evaluated as often as possible. Being usually battery powered, they consequently offer power-free parameters with regard to the aircraft system. On the other hand, their usage provides certain flexibility in the parameter requirement process after the freeze of the FTI architecture, as they can be implemented with limited impact on the wired FTI system. Last, and especially for the acquisition of rotating parameters, their integration can replace heavier systems based on slip ring or inductive transmission.

\section{Requirements Engineering}

The main key to match the size/weight/power consumption requirements as good as possible is to perform a smart, disciplined and focused requirements engineering process. Requirements engineering goes beyond the simple list of parameters to be acquired. For example, knowing the stakeholder for each analogue parameter (strain, pressure...) is one of the most important elements. The analogue parameters are indeed the main driver for defining a FTI architecture whereas bus parameter are less dimensioning.

It is obvious that the parameter with the lowest weight, the smallest size and the less consumed power is the one... which is not required! On the other hand, the most complex, most expensive parameter is the one, which has to be acquired in flight test!

Because of these unpretentiously facts, each parameter has to be challenged: is it really, really necessary to measure it in flight test or can it be acquired during bench testing? Often other MoC (Means of Compliance) like Laboratory Tests, Simulation or Calculation can be used to meet the requirements. The requirements review loops are very time consuming must be done not only once but repeated in iterative steps, with all stakeholders and always have to be very detailed. If done successfully, it leads to the following points which are essential for the success of a project:

- a realistic planning of time and cost is possible.

- The chosen FTI architecture meets the real requirements. Uncertainties can be minimized

- It provides a good visibility of the progress during the requirements engineering phase.

As it can be seen as an example in Fig 4, the requirements engineering process has to be initiated very early and has to be finished at depending on project size and complexity approximately one year before the First Flight. 


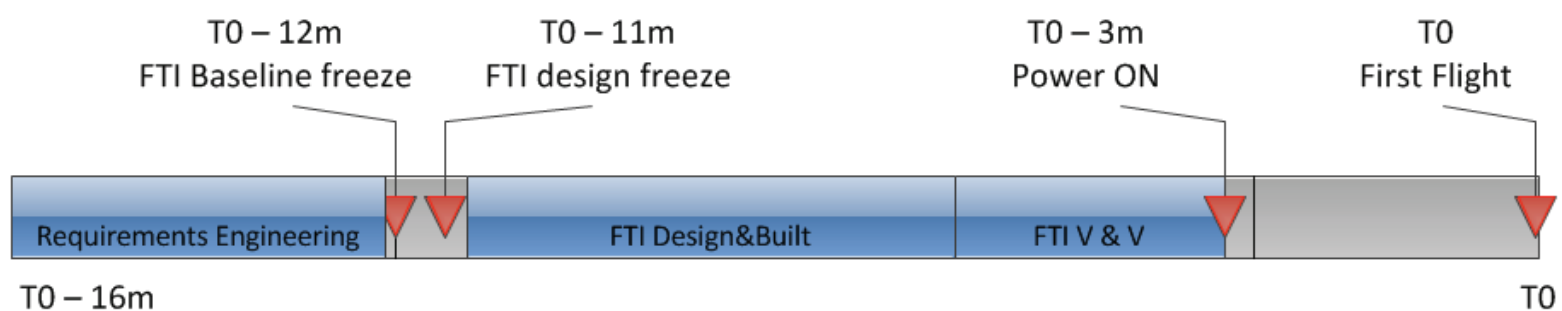

Fig 4 FTI process timeline

\section{Drawbacks and Limits}

By following all the steps mentioned above, some drawbacks have to be considered as well. Implementing new technologies and conceptscan bring certain risks. Risk assessment is mandatory to mitigate the possible negative impacts. Risk mitigation actions can be backup-scenarios, lab testing.

Following the rules mentioned in this article, it has to be noticed that flexibility, being one of the standard significant performance indicator for FTI systems, decreases to near zero. Avoiding cut-off connector and spare cabling, de-centralizing the installation clearly and drastically limit the possibilities to react to changed requirements.

It also has to be kept in mind that the FTI platform which will be integrated in such a demanding project will not be re-usable for other purposes. Brackets, trays, mechanical adaptations are customized for the specific needs. They can't be used anymore after the project has ended.

\section{Summary}

This paper discussed the different contribution factors which have to be adjusted in order to meet the requirements for FTI systems used in demonstrator aircraft platforms.

Some key elements were highlighted, with their potential benefits but also the risks which come along with their implementation.

This document showed also that not only technical aspects must be taken into account when designing a new type of such FTI architecture, but also organizational tasks. 